\title{
The effect of concentration and duration of normobaric oxygen in reducing caspase- 3 and -9 expression in a rat-model of focal cerebral ischaemia
}

\author{
Suyan Chen ${ }^{\mathrm{a}}$, Feng Gao ${ }^{\mathrm{c}, *}$, Anne Rowat ${ }^{\mathrm{b}}$, Huizhen Peng ${ }^{\mathrm{a}}$, Peng Wang ${ }^{\mathrm{a}}$, Zhenxiang Zhang, ${ }^{\mathrm{a},{ }^{*}}$ \\ Weihong Zhang ${ }^{\mathrm{a}}$, Xianyuan Wang ${ }^{\mathrm{d}}$, Lixia $\mathrm{Qu}^{\mathrm{a}}$
}

Short title: The effect of normobaric oxygen in a rat model of cerebral ischaemia

\author{
${ }^{\mathrm{a}}$ Department of Medical Nursing, School of nursing, Zhengzhou University, Zhengzhou, Henan, China \\ ${ }^{\mathrm{b}}$ School of Nursing, Midwifery \& Social Care, Edinburgh Napier University Edinburgh, Scotland \\ ${ }^{\mathrm{c}}$ Department of Neuroimmunology, Henan academy of Medical and Pharmaceutical Sciences, Zhengzhou \\ University, Zhengzhou, Henan, China \\ ${ }^{\mathrm{d} D e p a r t m e n t}$ of pathology, The second affiliated hospital, Zhengzhou University, Zhengzhou, Henan, China
}

\section{ABSTRACT}

The aim of this study was to determine the effect of different concentrations of normobaric oxygen (NBO) on neurological function and the expression of caspase-3 and -9 in a rat model of acute cerebral ischaemia. Sprague-Dawley rats ( $\mathrm{n}=120$ ) were randomly divided into four groups ( $\mathrm{n}=30$ per group), including 3 groups given NBO at concentrations of $33 \%, 45 \%$ or $61 \%$ and one control group given air (21\% oxygen). After 2 hours of ischaemic occlusion, each group was further subdivided into six subgroups $(n=5)$ during reperfusion according to the duration $(3,6,12,24,48$ or 72 hours) and concentration of NBO (33\%, $45 \%$ or $61 \%)$ or air treatment. The fluorescence quantitative Polymerase Chain Reaction (PCR) and immunohistochemistry were used to detect caspase-3 and -9 mRNA and protein relative expression respectively. The Neurologic Impairment Score (NIS) was significantly lower in rats given $61 \% \mathrm{NBO} \geq 3$ hours after reperfusion when compared to the control group $(P<0.05$, Mann-Whitney U). NBO significantly reduced caspase-3 and -9 mRNA and protein expression when compared to the control group at all NBO concentrations and time points ( $P<0.05$, ANOVA). The expression of caspase-3 and -9 was lower in the group given 61\% NBO compared any other group, and this difference was statistically significant when compared to the group given $33 \% \mathrm{NBO}$ for $\geq 48$ hours and the control group (both $P<0.05$, ANOVA). These findings indicate that NBO may inhibit the apoptotic pathway by reducing caspase-3 and -9 expression, thereby promoting neurological functional recovery after stroke.

Keywords: Normobaric oxygen; ischaemic rat model; Cerebral Ischaemic-Reperfusion; Apoptosis

\footnotetext{
${ }^{*}$ Correspondence to: Department of Medical Nursing, School of nursing, Zhengzhou University, Zhengzhou450001, Henan, China. Fax:+86037186565001. E-mail address: zhangzx6666@126.com(Z. Zhang) ${ }^{*}$ Correspondence to: Department of Neuroimmunology, Henan academy of Medical and Pharmaceutical Sciences, Zhengzhou University, Zhengzhou450052, Henan, China. E-mail address: gaoyuanshan@126.com (F. Gao)
} 


\section{Introduction}

Stroke is a major international health problem and a leading cause of death and disability in developed countries (Donnan et al, 2008). In Western countries approximately 80\% of strokes are ischaemic, whereas in China around 69\% are ischaemic compared to haemorrhagic strokes (Go et al., 2013; Hill., 2009; Li et al., 2008). Insufficient blood supply can result in brain ischaemia leading to white matter and grey matter cell death. It is clear that an increase in oxygen supply to the anoxic region can reduce the extent of neurological cell death (Sunami et al., 2000). Indeed, the principle aim of treating brain ischaemia in clinical practice is to improve blood circulation to the brain and increase blood and oxygen to the ischaemic penumbra, a vulnerable but potentially viable area of damaged brain cells surrounding the stroke core (Heiss and Graf., 1994).

As a clinical treatment hyperbaric oxygen (HBO) has been proposed to improve brain metabolism, reduce blood brain barrier permeability, brain oedema, intra-cranial cerebral perfusion, neuroplasticity and apoptosis both in animals and humans with ischaemic stroke (Efrati et al., 2013; Hu and Wang., 2013). However, HBO can also result in oxygen toxicity and the high pressure can cause damage to ears, eyes, lungs and increase the risk of pneumothorax and air embolism. HBO also requires hyperbaric chambers that cannot be easily used in acute stroke patients' given thrombolysis and require intensive monitoring. In comparison, normobaric oxygen (NBO) is less expensive, convenient to use, and is often routinely equipped at the patient's bedside without special conditions. A recent randomized controlled trial suggests that acute stroke patients given NBO at concentrations $<33 \%$ started within 24 hours of hospital-admission and given for 72 hours may slightly improve neurological recovery at 1 week (Roffe et al., 2011). However, a quasi-randomized study conducted by Ronning and Guldvog (1999) found that when $<33 \%$ NBO is given to non-hypoxic patients with minor or moderate strokes it may actually increase mortality. As such controversies still remain, oxygen supplementation is not recommended as a routine treatment in either Chinese or International stroke guidelines, unless oxygen saturation is $<95 \%$ or patients have clinical signs of hypoxia (National Stroke Foundation., 2010).

It has been suggested the giving NBO at high concentrations may be more beneficial to the survival of the ischaemic penumbra. Several studies have shown that NBO treatment given at concentrations of $90 \%$ to $100 \%$ in rat models of transient focal cerebral ischaemia can reduce the size of the cortical infarct (Liu et al., 2006; Singhal et al., 2002; Yuan et al., 2010). Furthermore, brief episodes of 100\% NBO does not appear to promote neurological damage in experimental stroke (Sun et al., 2014). High concentrations of HBO and/or NBO (80\%-100\%) have also been associated with reducing the occurrence of apoptosis via the cysteine-aspartic proteases (caspase) dependent apoptotic pathways (Akpan and Troy., 2013; Singhal and Lo., 2008). It is known that hypoxia can reduce adenosine-triphosphate (ATP) and release cytochrome C (Akpan and Troy., 2013). The latter is a caspase activating cofactor, which can promote cell death (Cho and Toledo-Pereyra., 2008). It has been suggested that high concentrations of oxygen could slow down death of the ischaemic cells in the penumbra region by inhibiting this caspase-apototic pathway (Singhal and Lo., 2008). Of the 8 types of caspases associated with ischaemic stroke, caspase-3 and -9 expression are found in rat controls (Geng et al., 2013; Zhang et al., 2004; Zhang et al., 2010). Caspase-3 is an important cysteine protease in the cell apoptosis process of the caspase cascade "waterfall”, and the effector molecule of performing DNA fragmentation that can induce cell apoptosis directly. Caspase- 9 is activated by an apoptosis protease activator -1 , a compound that is formed with cytochrome C. The activated caspase- 9 then cleaves and 
activates caspase-3 thereby inducing the cell to enter the irreversible death process (Sugawara et al., 2004). Recent research suggests that this process may be reversed with high concentrations of 95\%NBO to reduce brain swelling in ischaemic stroke rat models (Jin et al, 2014). However, there are safety concerns with giving very high concentrations of NBO (80\% to $100 \%)$ as it can lead to hyperoxia, resulting in acute lung injury, toxicity and poor outcomes after stroke (Cornet et al, 2013; Huang et al., 2009; Zhou et al., 2006).

The aim of this study is to determine whether NBO given at medium concentrations (33\%, 45\%, $61 \%$ ), which may be better tolerated by stroke patients, and given over various time points could reduce neurological damage and caspase-3 and/or caspase-9 expression (i.e. apoptosis) in a rat model of acute brain ischaemia. 


\section{Results}

2.1. $\quad$ NBO improved Neurologic Impairment Scores

During reperfusion the rats' nerve function were checked prior to and after NBO or air treatment via Neurologic Impairment Scores (NIS) assessment. Before NBO treatment the median NIS = 2 in all 24 groups. Table 1 shows that the median NIS after NBO treatment was lower in rats receiving 33\% NBO $\geq 72$ hours, $45 \%$ NBO $\geq 6$ hours and $61 \%$ NBO $\geq 3$ hours when compared to baseline measurements (1 versus 2). Compared to the control group, the NIS was lower at $45 \%$ and $61 \%$ NBO at $\geq 48$ hours ( $P=0.022$, Kruskal Wallis) and in all NBO treatment groups at 72 hours ( $P=0.018$, Kruskal Wallis)

\subsection{NBO reduced caspase-3 and caspase- 9 mRNA and protein expression}

The mRNA expression levels of caspase-3 and -9 in brain at different time points in all groups were checked via Real-time Fluorescent Quantitative PCR analysis. The mRNA expression of caspase-3 (Fig.1a) and caspase-9 (Fig.1b) were significantly lower in NBO treatment groups when compared to the control group regardless of NBO concentration or duration (all $P<0.01$, ANOVA). The mRNA expressions for caspase-3 and -9 were lower in the group given 61\% NBO compared to 33\% and $45 \%$ NBO, but it was statistically significant when compared to the group given $33 \%$ NBO, regardless of duration (all $P<0.05$, ANOVA).

The protein expression levels of caspase-3 and -9 in the ischaemic penumbra in the control group and NBO treatment groups were determined via indirect immunohistochemical assay. The localization of caspase in the ischaemic penumbra was indicated by brown staining (Fig.2a, Fig.3a). The protein expression of caspase-3 and -9 positive cells gradually reduced at all concentrations of NBO (Fig.2b, Fig.3b) and these differences were statistically significant when compared to the control group (all $P<0.05$, ANOVA). Comparing the treatment groups, protein expression was significantly lower at $61 \%$ NBO compared to $33 \%$ NBO, regardless of NBO duration $(P<0.05$, ANOVA). 


\section{Discussion}

The results of this study found that in all NBO treatment and the control groups, caspase-3 and -9 mRNA and protein expression increased in the ischaemic rat brain over time (3 to 72 hours). However, medium concentrations of NBO reduced the level of caspase- 3 and -9 at all time points and was lowest in the group given 61\% NBO. Interestingly, we also found that neurological impairment was lower at earlier time points as NBO concentration increased. In particular we found that the lowest NIS occurred in the $61 \%$ NBO group from 3 to 72 hours. This was noticeably different to the control group (oxygen 21\%) who had the highest levels of caspase -3 and -9 and worsening of the neurological impairment over time. Therefore, these results support the view that neuroprotection can be achieved by increasing concentrations of oxygen to the ischaemic penumbra (Singhal., 2006). Our results also suggest that longer NBO exposure at medium concentrations may yield some benefit to improving neurological recovery after stroke, but whether it is sustainable due to the evolving apoptosis processes still remains to be determined.

By using a rat model of focal cerebral ischaemia we have been able to provide further understanding of the apoptosis pathway after stroke. A previous study found that giving 95\% NBO therapy in an ischaemic rat model reduced caspase-9, but they did not find a significant reduction in caspase-3 (Jin et al., 2014). However, rats were exposed to NBO for only 90 minutes during middle cerebral artery occlusion, which may not have been long enough to detect the reduction in caspase -3 , which occurs downstream from caspase-9 after the cytochrome c-dependent cascade is initiated (Sugawara et al., 2004). It is also difficult to compare our results to previous experimental studies due to the differences in the initiation and exposure to NBO treatment after cerebral ischaemia. Indeed, the majority of these studies have focused on giving NBO at very high concentrations (80-100\%) for shorter durations (2-12 hours) (Singhal et al., 2002, 2006; Liu et al., 2006; Esposito et al., 2013). However, there are concerns that very high concentrations of NBO will generate oxygen free radicals causing worsening of the neurological injury (Cornet et al., 2013). Certainly, a recent randomized controlled trial that investigated the effects of high flow (30-45 litres per minute) NBO (100\%) given to acute stroke patients for 8 hours was terminated early due to excess mortality in the NBO group when compared to the group on air/low flow oxygen (40\% versus 17\%) (Singhal., 2014). However, preliminary findings from the largest NBO stroke trial to date, found no benefit with lower concentrations of NBO (24-32\%) given up to 72 hours in 8000 acute stroke patients (Roffe et al., 2014 a, b). Thus medium concentrations of $61 \%$ NBO may be safer and better tolerated by acute stroke patients than high NBO concentrations, but are possibly more effective than lower NBO concentrations at reducing short-term neurological impairment. However, its efficacy particularly in terms of improving longer-term outcomes would need to be confirmed in a clinical trial.

There are a number of limitations. Firstly, experimental studies are limited by small sample sizes ( $\mathrm{n}=5$ per group), however we were able to detect statistically significant differences between the experimental groups and the control. Second, rat models of focal cerebral ischaemia are subject to tightly controlled laboratory conditions and include young healthy animals, which often limits its applicability to stroke patients (Casals et al., 2011). Third, the timing of NBO delivery was started 2 hours after ischaemia/reperfusion, whereas in contrast oxygen is not usually started for up to 6-72 hours after stroke onset in human studies (Ronning et al., 1999; Padma et al., 2010; Singhal., 2014; Roffe., 2014a). Fourth, we did not measure the apoptosis effects of NBO once it was discontinued, but 
there is evidence to suggest that the benefits of NBO to salvageable tissue may be lost within 24 hours if discontinued (Henniger et al, 2006). Finally, it must be acknowledged that caspase -3 and -9 are not absolute in the apoptosis pathway and apoptosis may progress even when caspases are inhibited (Ferrer et al, 2003). Therefore, we recommend that future studies of caspases are considered in conjunction with other apoptosis assays common after stroke.

In conclusion, the benefits of NBO on caspase -3 and -9 are dependent on factors such as the concentration and duration of NBO, which appears to have neuroprotective benefits for ischaemia-reperfusion injury in rat brain. We also feel that further experimental research is warranted to ensure the design of safe and effective randomized controlled trials for stroke patients. Future studies should pay particular attention to the timing of NBO delivery after stroke onset/reperfusion status and the longer-term effects of hyperoxia on the apoptosis pathway once NBO is discontinued. 


\section{Experimental Procedures}

4.1 Animals

Adult Male specific pathogen free (SPF) level Sprague-Dawley rats ( $n=120,250 \sim 300$ g) were used for this study (experimental animal center of Zhengzhou University, license No.: SCXK (Henan)

2010-0002). They were housed in SPF laboratory cages in a controlled environment $\left(22.0 \sim 24.0^{\circ} \mathrm{C}\right)$ and were maintained under a 12/12 h light/dark cycle with free access to food and water. The "Laboratory Animal Care and Use Committee” at the University of Zhengzhou approved all experimental protocols.

\subsection{Experimental focal cerebral ischaemia and Neurologic Impairment Scores (NIS)}

The rats were anesthetized with $10 \%$ chloral hydrate $(0.35 \mathrm{ml} / 100 \mathrm{~g})$ by intra-peritoneal injection. A heating pad was used to maintain the rats’ body temperature at $37 \pm 0.5^{\circ} \mathrm{C}$. An intraluminal suture blocked the origin of the middle cerebral artery, occluding blood flow from the internal carotid artery (Longa et al., 1989). The incision was closed, leaving $1 \mathrm{~cm}$ of the suture protruding so it could be withdrawn to allow reperfusion 2 hours later. All rats’ neurologic examinations were scored prior to and after NBO/air using a five-point scale: 0 = no neurological deficits; 1 = failure to extend left forepaw fully; 2 = circling to the left; 3 = falling to the left; 4 = no spontaneous walking and loss of consciousness (Longa et al., 1989).

\subsection{Experiment groups and NBO treatment}

The 120 rats were randomly allocated to three main experimental groups and exposed to $33 \%$, $45 \%$ and $61 \%$ NBO respectively or the control group that was exposed to room air only ( $n=30$ per group).

After 2 hours of ischaemic occlusion, each group was further subdivided into six subgroups ( $\mathrm{n}=5)$ and given NBO/air for either 3, 6, 12, 24, 48 or 72 hours during reperfusion. NBO/air was given to the rats in a closed chamber $(50 \mathrm{~cm} \times 30 \mathrm{~cm} \times 30 \mathrm{~cm})$ with two $1 \mathrm{~cm}$ holes connected to the outside, one side with oxygen cylinder and nitrogen cylinder, and the other with the oxygen sensor used to monitor the oxygen concentration in the chamber.

\subsection{Real-time Fluorescent Quantitative polymerase chain reaction (PCR)}

At the end of their NBO or air treatment, the rats in each group were deeply anesthetized with excessive $10 \%$ chloral hydrate. The brain of the rats were removed and placed into liquid nitrogen for the analysis of Fluorescent Quantitative PCR. Fluorescence quantitative PCR primers, fluorescent dye and reference GAPDH was synthesized using RNase-free DNase 1 (TaKaRa limited company, Dalian, China). Following the manufacturer's protocol, total RNA was prepared from the ischaemic tissue using the TRI®zol RNA isolation kit (Invitrogen, Sweden). The RNA was then resuspended in $10 \mu$ of nuclease free water and was measured at 260 and $280 \mathrm{~nm}$ using a spectrophotometer (Eppendorf PhysioCare Concept, Germany), All values were within 1.8-2.0, with no genomic DNA contamination. The RNA were adjusted for transcription of cDNA (Takara biotechnology, Dalian). The target gene information is in table 2 .

The cDNA for the real-time PCR reactions was created in a total volume of $20 \mu$ as per the manufacturer's instructions (TaKaRa biotechnology, Dalian, China). Amplification reactions were performed using a 7500 fast Real-Time PCR System (Applied Biosystems, Massachusetts, USA) using the following cycling conditions: 1 cycle at $95{ }^{\circ} \mathrm{C} 30$ seconds (s); 40 cycles at $95{ }^{\circ} \mathrm{C} 3 \mathrm{~s}$ and $60{ }^{\circ} \mathrm{C} 30 \mathrm{~s} ; 1$ 
cycle at $95{ }^{\circ} \mathrm{C} 15 \mathrm{~s}, 60{ }^{\circ} \mathrm{C} 1 \mathrm{~min}, 95{ }^{\circ} \mathrm{C} 15 \mathrm{~s}$ and $60{ }^{\circ} \mathrm{C} 15 \mathrm{~s}$. We calculated the average PCR threshold cycle (Ct) values of three repeated reference and objective genes of each sample, then calculated the relative quantitative $2^{-\triangle \Delta \mathrm{Ct}}$ to analyze relative gene expression [ $\triangle \triangle \mathrm{Ct}=$ (target gene Ctinternal reference

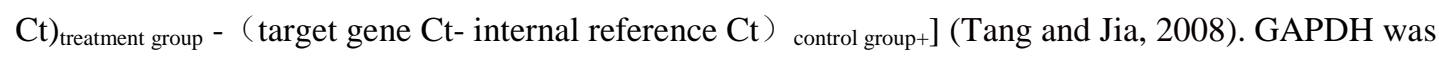
taken as internal reference, and the expression of control group at 3 hours was defined as 1 .

\subsection{Immunohistochemistry}

The brain tissues were cut into coronal sections ( $4 \mu \mathrm{m}$ thickness), They were incubated in rabbit anti-caspase-3 or anti-caspase-9 polyclonal antibody (BA2142, BA0690 1:100, Wuhan Boster Biotechnology, Wuhan) overnight at $26^{\circ} \mathrm{C}$. Immunostaining was revealed with streptoavidin -biotin complex (SABC, Wuhan Boster Biotechnology, Wuhan) and a chromogenic agent 3, 3'-diaminobenzidine (DAB, Wuhan Boster Biotechnology, Wuhan). We sequentially chose 5 visual fields in the ischemic penumbra of each rat at $400 \times$ magnification (Nikon Eclipse TS 100, Nikon Instruments, Japan). The number of caspase-3 or -3 positive cells in each field was quantified by the image analysis system (NIS-Elements BR 3.2, Nikon Instruments, Japan).

4.6 Statistical analysis

Data were analysed using SPSS for windows (version 12 SPSS Inc, Chicago, IL. 2004). Neurological function scores were expressed as median (interquartile range, IQR). The immunohistochemical positive cells numbers and $2^{-\Delta \Delta \mathrm{Ct}}$ were expressed as means \pm standard deviations. The differences between groups were calculated using Kruskal Wallis and Mann Whitney U for non-paramentric comparisons and ANOVA and unpaired t-test for parametric comparisons. $P$-values $<0.05$ were considered statistically significant.

Acknowledgement This project was supported by Henan Province Science and Technology Department (Item number: 122300410082), and the Henan Province Education Department 5451 foundation (Item number: 201201065).

Conflict of interest: The authors declare that they have no conflict of interest. 


\section{References}

Akpan, N., Troy, C.M., 2013. Caspase Inhibitors: Prospective Therapies for Stroke. Neuroscientist. 19, 129-136.

Casals, J.B., Pieri, N.C., Feitosa, M.L, Ercolin, A.C., Roballo, K.C., Barreto, R.S., Bressan, F.F., Martins, D.S., Miglino, M.A., Ambrósio, C.E., 2011.The Use of Animal Models for Stroke Research: A Review. Comp Med. 61, 305-313.

Cho, B.B., Toledo-Pereyra, L.H., 2008. Caspase-independednt programmed cell death folowing ischemic stroke. J Invest Surg. 21, 141-147.

Cornet A., Kooter A.J., Peters M.J.L, Smulders Y.M., 2013 The potential harm of oxygen therapy in medical emergencies. Crit Care. 17, 313 doi:10.1186/cc12554

Donnan, G., Fisher, M., Macleod, M., 2008. Stroke. Lancet. 371, 1612-1623.

Efrati, S., Fishlev, G., Bechor, Y., Volkov, O., Bergan, J., Kliakhandler, K., Kamiager, I., Gal, N., Friedman, M., Ben-Jacob, E., Golan, H., 2013. Hyperbaric oxygen induces late neuroplasticity in post stroke patients -randomized, prospective trial. PLoS One. 8, e53716.

Esposito, E., Mandeville, E.T. Hayakawa, K., Singhal, A.B., 2013. Effects of normobaric oxygen on the progression of focal ischemia in rats. Exp Neurol. 249, 33-38.

Ferrer, I., Friguls, B., Dalfó, E., Justicia, C., Planas, A.M., 2003. Caspase-dependent and caspase-independent signalling of apoptosis in the penumbra following middle cerebral artery occlusion in the adult rat. Neuropathol Appl Neurobiol. 29, 472-81.

Geng, X.K., Parmar, S., Li, X.M., Peng, C.Y., Ji, X.M., Chakraborty, T., Li, W.A., Du, H.S., Tan, X.M., Ling, F., Guthikonda, M., Rafols, J.A., Ding, Y.C., 2013. Reduced apoptosis by combining normobaric oxygenation with ethanol in transient ischemic stroke. Brain Res. 1531, 17-24.

Go, A.S., Mozaffarian, D., Roger, V.L., Benjamin, E.J., Berry, J.D., Borden, W.B., Bravata, D.M., Dai, S., Ford, E.S., Fox, C.S., Franco, S., Fullerton, H.J., Gillespie, C., Hailpern, S.M., Heit, J.A., Howard, V.J., Huffman, M.D., Kissela, B.M., Kittner, S.J., Lackland, D.T., Lichtman, J.H., Lisabeth, L.D., Magid, D., Marcus, G.M., Marelli, A., Matchar, D.B., McGuire, D.K., Mohler, E.R., Moy, C.S., Mussolino, M.E., Nichol, G., Paynter, N.P., Schreiner, P.J., Sorlie, P.D., Stein, J., Turan, T.N., Virani, S.S., Wong, N.D., Woo, D., Turner, M.B., 2013. Heart disease and stroke statistics - 2013 update: a report from the American Heart Association. Circulation. 127, e6-e245.

Henniger, N., Sicard, K.M., ScHmidt, K.F., Fisher, M., 2006, N Normobaric hyperoxia delays perfusion/diffusion mismatch evolution, reduces infarct volume, and differentially affects neuronal cell death pathways after suture middle cerebral artery occlusion in rats. Stroke. 37, 1283-1287.

Heiss, W.D., Graf, R., 1994, The ischemic penumbra. Curr Opin Neurol. 7, 11-19.

Hill, C., 2009. Death registrations in England and Wales, 2008, causes. Health. Stat. Q. 43, 68-76.

Hu, P., Wang, A., 2013. Study of effects of hyperbaric oxygen on the treatment of acute ischemic cerebral stroke. China Mod Med. 20, 55-56.

Huang, D., Fang, F., Xu, F., 2009. The role of expression of Toll-like receptor 2 and Toll-like receptor 4 in hyperoxia-induced acute lung injury in rat. China Crit Care Med. 21, 644-647. 
Jin, X.C., Liu, J., Liu, K.J., Rosenberg, G.A., Yang, Y., Liu, W.L., 2014. Normobaric hyperoxia combined with minocycline provides greater neuroprotection than either alone in transient focal cerebral ischemia. Exp Neurol. 240, 9-16.

Li, W., Liu, M., Feng, S., Li, W., Wu, B., Fang, Y., Wang, L., Zhao, S., Zhao, M., Zhang, S., 2008. Clinical characteristics and long-term prognosis of patients with ischemic and hemorrhagic stroke. Natl Med J China. 88, 892-897.

Liu, S., Liu, W., Ding, W., Miyake, M., Rosenberg, G.A., Liu, K.J., 2006. Electron paramagnetic resonance-guided normobaric hyperoxia treatment protects the brain by maintaining penumbral oxygenation in a rat control of transient focal cerebral ischaemia. Journal of Cerebral Blood Flow \& Metabolism. 26, 1274-1284.

Longa, E.Z., Weinstein, P.R., Carlson, S., Cummins, R., 1989. Reversible middle cerebral artery occlusion without craniectomy in rats. Stroke. 20, 84-91.

National Stroke Foundation, 2010. Acute medical and surgical management. In: Clinical guidelines for stroke management 2010., Vol.,ed., Melbourne(Australia), pp. 58-67.

Roffe, C., Ali, K., Warusevitane, A., 2011. The SOS pilot study: a RCT of routine oxygen supplementation early after acute stroke-effect on recovery of neurological function at one week. Plos One. 6, e19113.

Roffe, C., Nevatte, T., Crome, P., Sim, J., Pountain, S., Handy, L., Handy, P., 2014a. The Stroke Oxygen Study (SO2S) - a multi-center,study to assess whether routine oxygen treatment in the first 72 hours after a stroke improves longterm outcome: study protocol for a randomized controlled trial. Trials. 15, 99.

Roffe, C., on behalf of the stroke oxygen study collaborative group., 2014b. Stroke oxygen Study Preliminary outcome presentation. Retrieved from: http://www.so2s.co.uk (accessed 1 May 2015)

Ronning, O., Guldvog, B., 1999. Should stroke victims routinely receive supplemental oxygen? A quasi-randomized controlled trial. Stroke. 30, 2033-2037.

Padma, M.V., Bhasin, A., Bhatia, R., Garg, A., Singh, M.B., Tripathi, M., Prasad, K., 2010.

Normbobaric oxygen therapy in acute ischemic stroke: a pilot study in Indian patients. Ann Indian Acad Neurol. 13, 284-288.

Singhal, A.B., Dijkhuizen, R.M., Rosen, B.R., Lo, E.H., 2002. Normobaric hyperoxia reduces MRI diffusion abnormalities and infarct size in experimental stroke. Neurol. 58, 945-52.

Singhal, A.B., 2006. Oxygen therapy in stroke: past, present, and future. Int J Stroke. 1,191-200.

Singhal, A.B., Lo, E.H., 2008. Advances in emerging nondrug therapies for acute stroke 2007. Stroke. 39, 289-291.

Singhal, A.B., on behalf of the SPOTRIAS Investigators., 2014. Normobaric oxygen therapy in acute ischemic stroke trial. Retrieved from:

http://www.strokecenter.org/trials/clinicalstudies/normobaric-oxygen-therapy-in-acute-ischemic-stroke -trial (accessed 1 May 2015).

Sun, L., Wolferts, G., Veltkamp, R., 2014. Oxygen therapy does not increase production and damage induced by reactive oxygen species in focal cerebral ischemia. Neurosci Lett. 577, 1-5. 
Sugawara, T., Fujimura, M., Noshita, N., Kim, G.W., Saito, A., Hayashi, T., Narasimhan, P., Maier, C.M., Chan, P.H., 2004. Neuronal death/survival signaling pathways in cerebral ischemia. NeuroRx. $1: 17-25$.

Sunami, K., Takeda, Y., Hashimoto, M., Hirakawa, M., 2000. Hyperbaric oxygen reduces infarct volume in rats by increasing oxygen supply to the ischemic periphery. Crit Care Med. 28, 2831-2836.

Tang, Y., Jia, Y., 2008. Method of Processing Real Time PCR Data. Biotechnol. 18, 89-91.

Yuan, Z.R., Liu, W.L., Liu, B.Y., Schnell, A., Liu, K.J., 2010. Normobaric hyperoxia delays and attenuates early nitric oxide production in focal cerebral ischemic rats. Brain Res. 1352, 248-254.

Zhang, F., Yin, W., Chen, J., 2004. Apoptosis in cerebral ischemia: executional and regulatory signaling mechanisms. Neurol Res. 26, 835-845.

Zhang, Z., Liu, L., Gong, X., Peng, X., Chen, B., 2010. Changes of Cathepsin D and caspase-9 Expression in Rats after Cerebral Ischemia-Reperfusion. Progress Mod Biomed. 10, 851-854.

Zhou, H., Pan, X., Wang, J., Ma, Y., Guo, C., 2006. The effect of rat different time inhalant normalbaric hyperxia at lung injury. Chin J Anesthesiol. 26, 473-475. 
Table 1: The Median (IQR) Neurologic Impairment Scores (NIS) for each group after NBO treatment compared to the control group ( $n=5$ per group)

\begin{tabular}{lllllll}
\hline Group & 3h & 6h & $12 \mathrm{~h}$ & $24 \mathrm{~h}$ & $48 \mathrm{~h}$ & $72 \mathrm{~h}$ \\
\hline $61 \%$ NBO & $1.0(1.0,2.0)^{*}$ & $1.0(1.0,1.5)^{*}$ & $1.0(1.0,2.0)^{*}$ & $1.0(0.5,2.0)^{*}$ & $1.0(0.5,2.0)^{*}$ & $1.0(0.5,1.5)^{*}$ \\
$45 \%$ NBO & $2.0(1.0,2.0)$ & $1.0(1.0,2.0)$ & $1.0(1.0,2.0)^{*}$ & $1.0(1.0,2.0)^{*}$ & $1.0(1.0,1.5)^{*}$ & $1.0(1.0,2.0)^{*}$ \\
$33 \%$ NBO & $2.0(1.5,2.0)$ & $2.0(1.0,2.0)$ & $2.0(1.0,2.0)$ & $2.0(1.0,2.0)$ & $2.0(1.0,2.0)^{*}$ & $1.0(1.0,2.0)^{*}$ \\
Control & $2.0(2.0,2.5)$ & $2.0(1.5,3.0)$ & $2.0(2.0,2.5)$ & $2.0(2.0,2.5)$ & $3.0(2.0,3.5)$ & $3.0(2.0,3.0)$ \\
\hline$x^{2}, d f=3$ & 5.612 & 5.278 & 5.900 & 5.949 & 9.587 & 10.052 \\
$P$ & 0.132 & 0.153 & 0.117 & 0.114 & $\mathbf{0 . 0 2 2}$ & $\mathbf{0 . 0 1 8}$ \\
\hline
\end{tabular}

Note: The differences between groups were calculated using Kruskal Wallis H test and paired comparisons using Mann-Whitney U test, $* \mathrm{P}<0.05$.

Abbreviations: df, degrees of freedom; IQR, Interquartile range; h, hours; NIS, Neurological Impairment Score; NBO, normobaric oxygen; $x^{2}$, Chi-square for H-test. 
Table 2: The gene primer sequence and product size used for GAPDH, caspase-3 and caspase-9.

\begin{tabular}{llll}
\hline Gene name & Oligonucleotides used for real-time PCR primer & $\begin{array}{c}\text { Product } \\
\text { size (bp) }\end{array}$ & $\begin{array}{c}\text { GenBank } \\
\text { Accession }\end{array}$ \\
\hline sequence 5' to 3' & F: CTGAGCCAGATGCTGTCCCATA & NM-031632.1 \\
& R: GACACCATCCAAGGTCTCGATGTA & & \\
caspase-3 & F:GAGACAGACAGTGGAACTGACGATG & 147 & NM-012922.2 \\
RAPDH & F: GGCGCAAAGTGACTGGATGA & & NM-017008.4 \\
& R: ATGGTGGTGAAGACGCCAGTA & & \\
\hline
\end{tabular}

Abbreviation: PCR, polymerase chain reaction (PCR); F, forward primer for conventional SYBRß Green real-time PCR; R, reverse primer for SYBR® Green real-time PCR. 
Fig.1 The expression of caspase-3 (A) and caspase-9 (B) mRNA in the control group (air, oxygen $21 \%)$ and NBO treatment groups $(33 \%, 45 \%, 61 \%)$ at all time points: $3 \mathrm{~h}, 6 \mathrm{~h}, 12 \mathrm{~h}, 24 \mathrm{~h}, 48 \mathrm{~h}, 72 \mathrm{~h}$ ( $n=5$ per group).

Data are expressed as mean $\pm \mathrm{SD}$, the differences between groups were calculated using ANOVA for group parametric comparisons; treatment groups were compared with the control group, $* P<0.01 ; 61 \%$ and 45\% NBO group compared with the 33\% NBO group, \#P $<0.05$.

Abbreviations: mRNA, Messenger RibonucleicAcid; NBO, normobaric oxygen; SD, Standard Deviation.

A

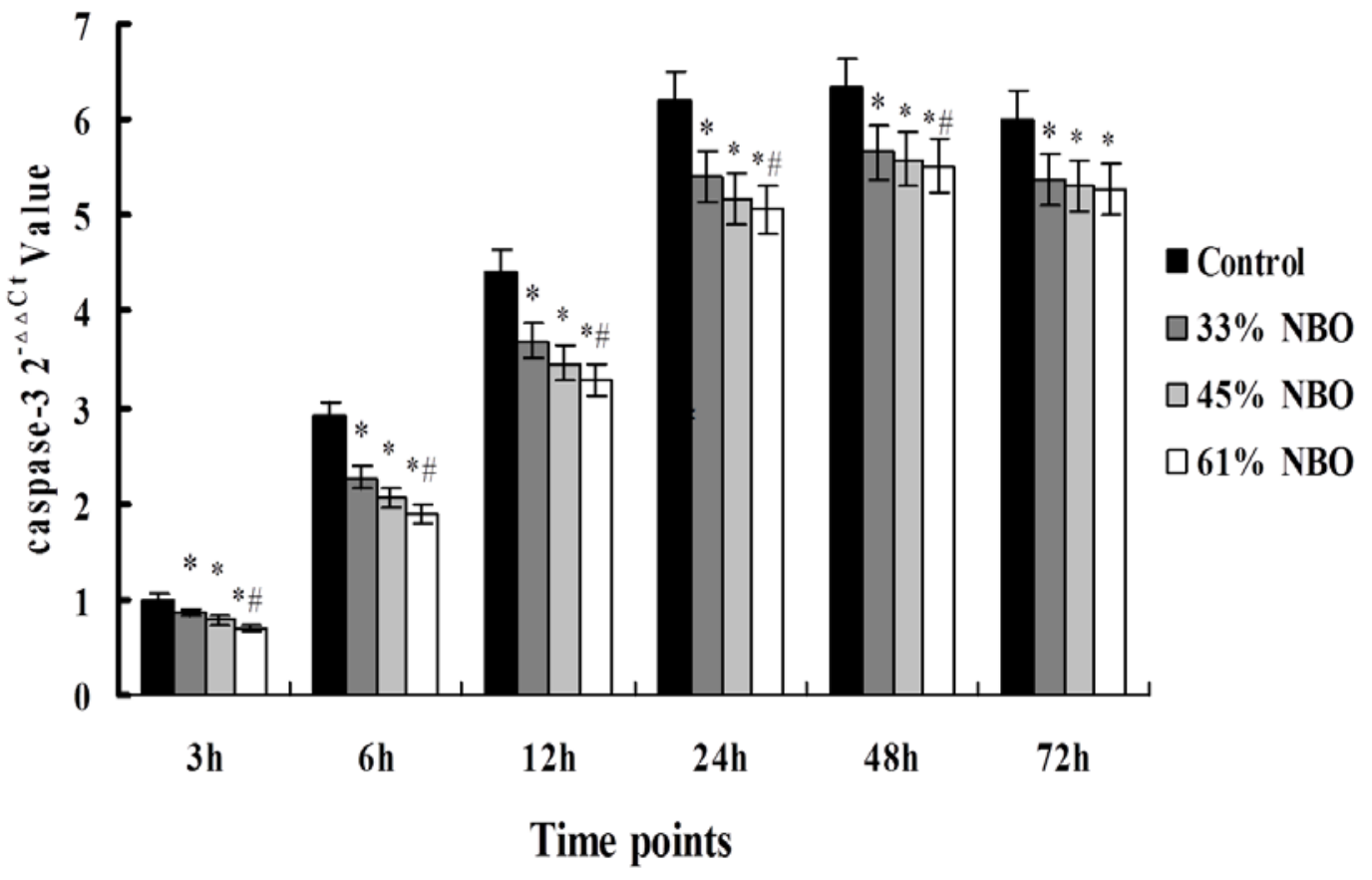




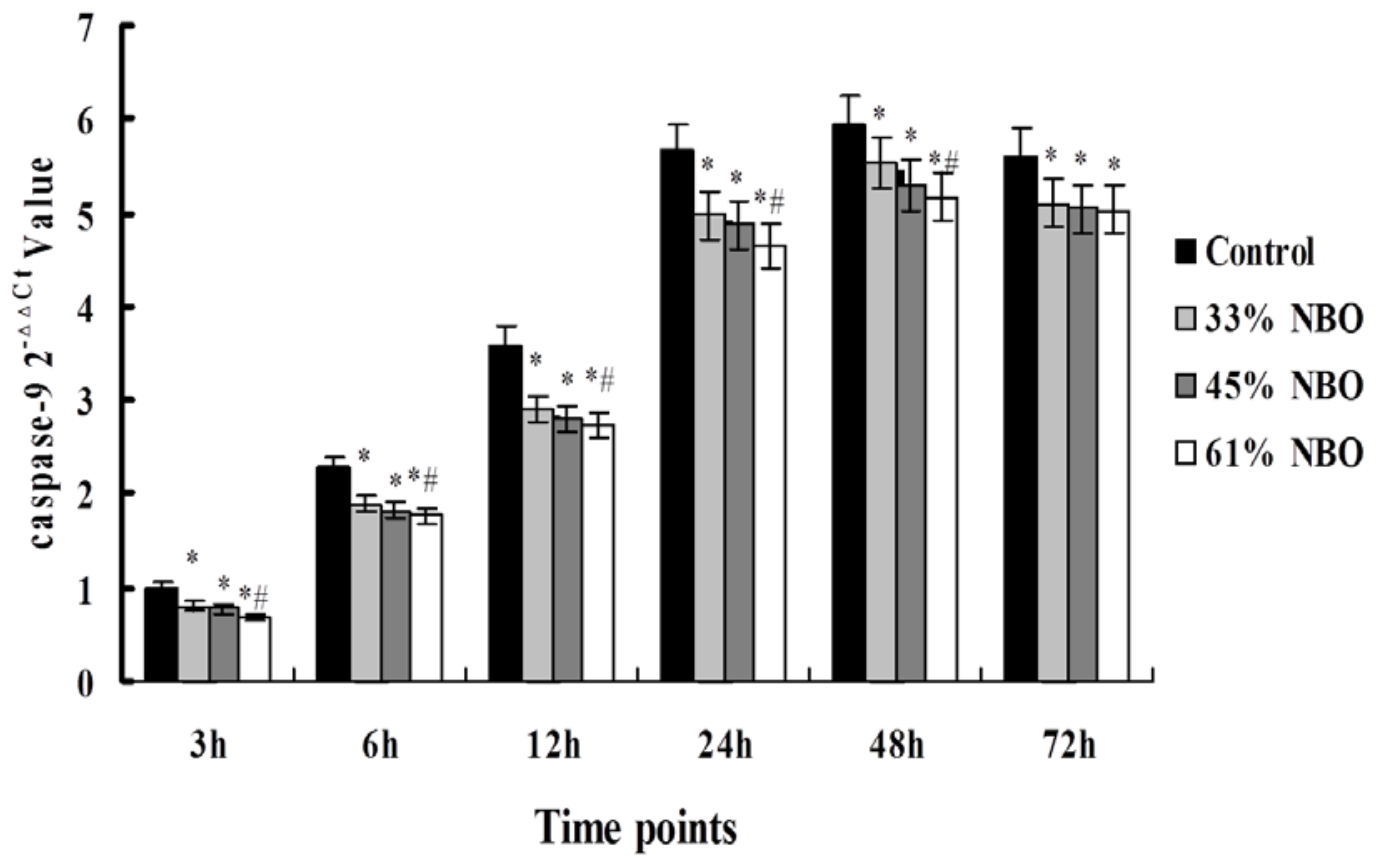


Fig.2 Immunohistochemistry analysis of caspase-3 for the control and NBO treatment groups. (A) Expression of caspase- 3 in rat brain tissue at all time points: $3 \mathrm{~h}, 6 \mathrm{~h}, 12 \mathrm{~h}, 24 \mathrm{~h}, 48 \mathrm{~h}, 72 \mathrm{~h}(\mathrm{n}=5$ per group). SABC stain $\times 400$ (Scale bar $200 \mu \mathrm{m}$ ). (B) The number of caspase-3 positive cells in control and NBO treatment groups ( $n=5$ per group).

Data are expressed as mean $\pm \mathrm{SD}$, the differences between groups were calculated using ANOVA for parametric comparisons; NBO treatment groups were compared with the control group, ${ }^{*} P<0.01$; $61 \%$ and $45 \%$ NBO group compared with the 33\% NBO group, \#P $<0.05$.

Abbreviations: NBO, normobaric oxygen; SABC, strept avidin-biotin complex.

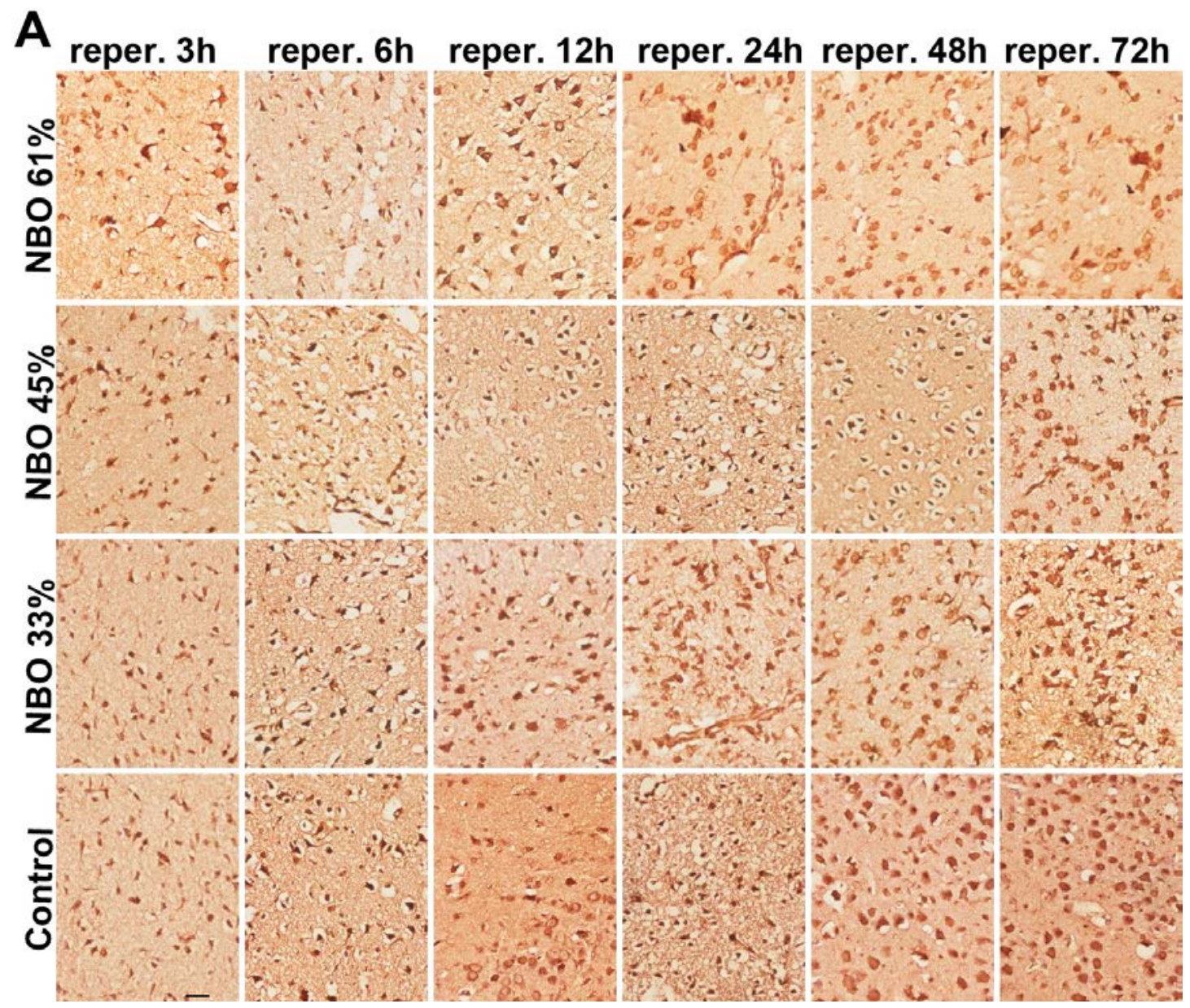




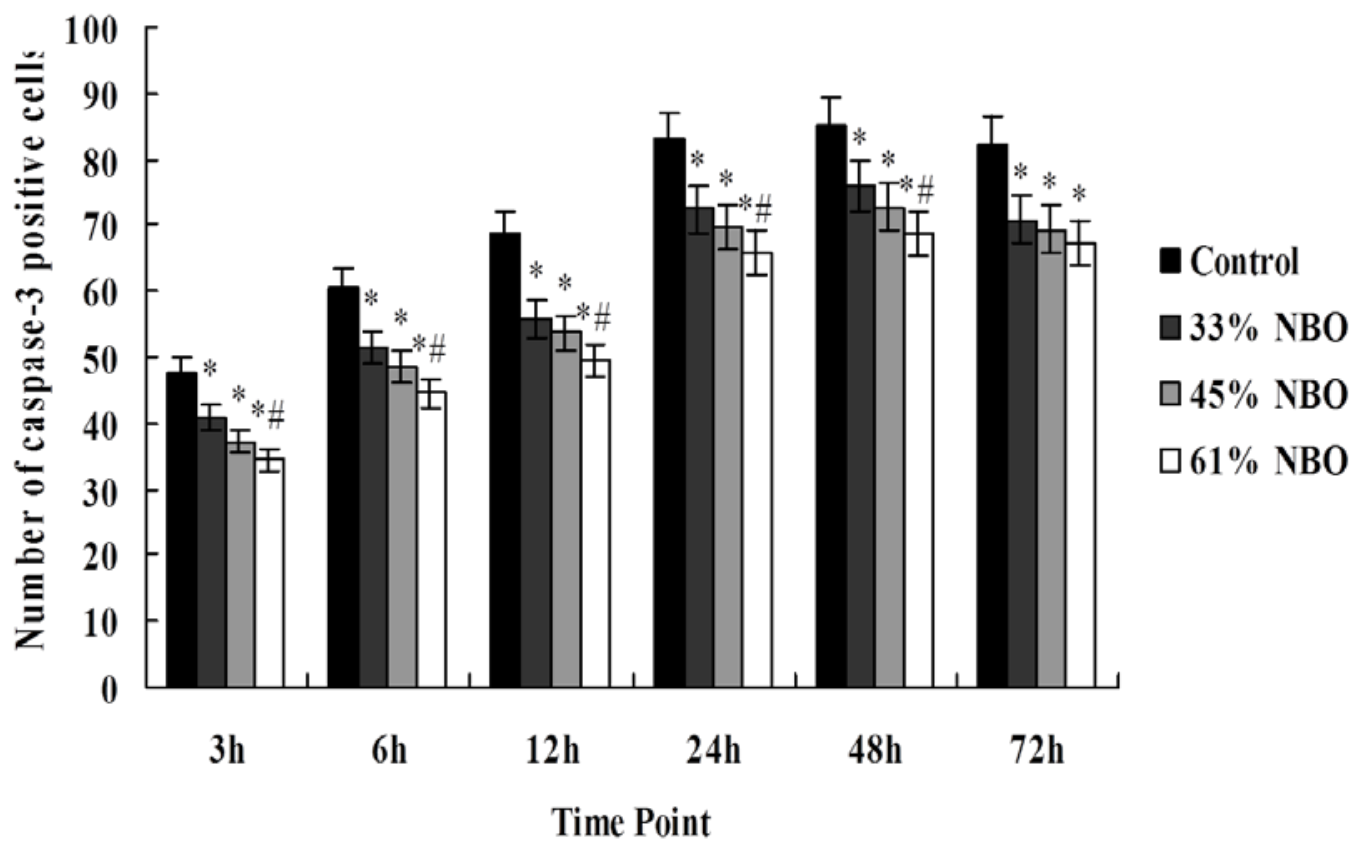


Fig.3 Immunohistochemistry analysis of caspase-9 for the control and NBO treatment groups. (A) Expression of caspase- 9 in rat brain tissue at all time points: $3 \mathrm{~h}, 6 \mathrm{~h}, 12 \mathrm{~h}, 24 \mathrm{~h}, 48 \mathrm{~h}, 72 \mathrm{~h}(\mathrm{n}=5 \mathrm{per}$ group) - SABC stain $\times 400$ ( Scale bar $200 \mu \mathrm{m}$ ). (B) The number of caspase-9 positive cells in control and NBO treatment groups ( $n=5$ per group).

Data are expressed as mean $\pm \mathrm{SD}$, the differences between groups were calculated using ANOVA for parametric comparisons; NBO treatment groups compared with the control group, ${ }^{*} P<0.01 ; 61 \%$ and 45\% NBO group compared with the 33\% NBO group, \#P $<0.05$.

Abbreviations: NBO, normobaric oxygen; SABC, strept avidin-biotin complex

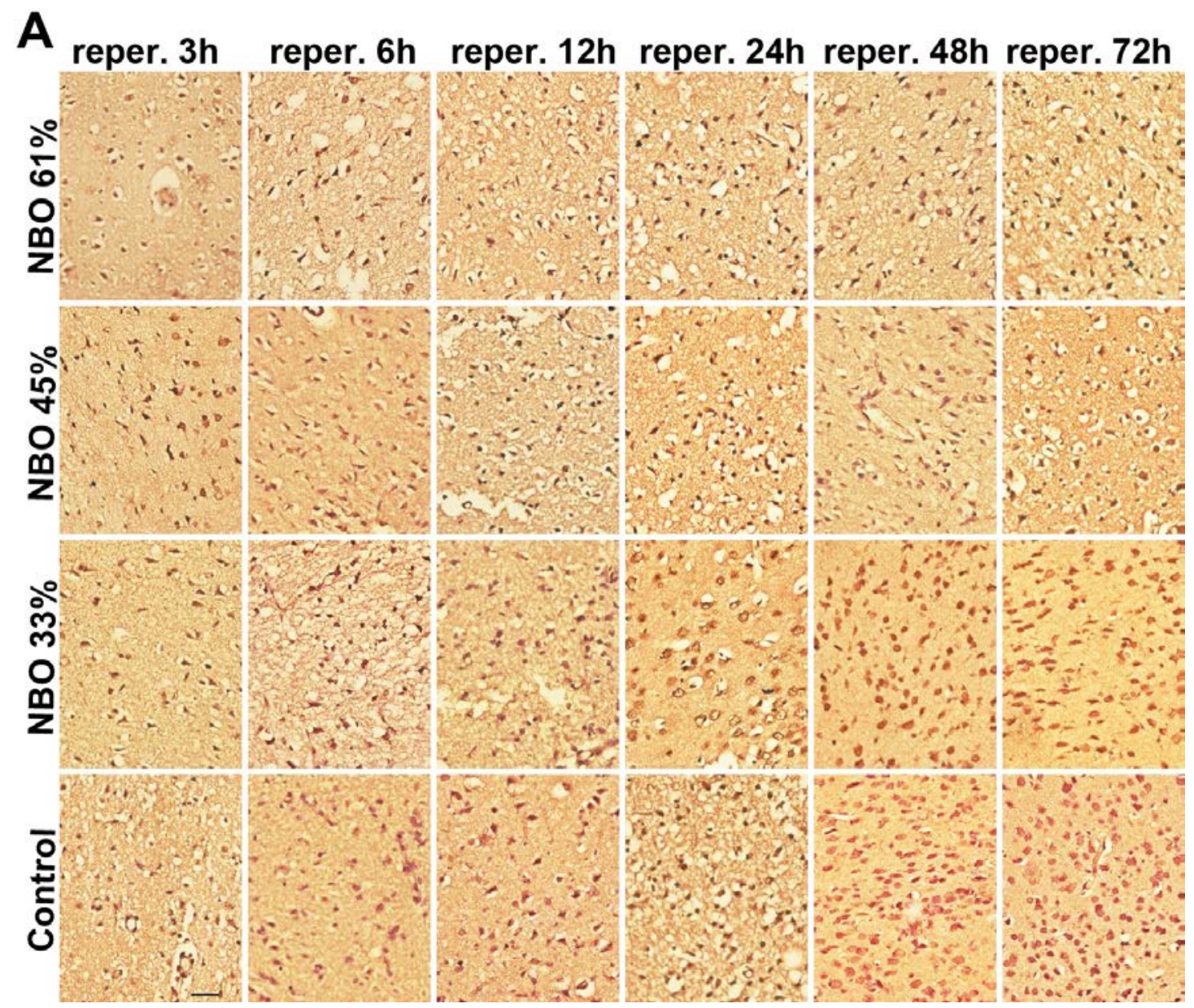




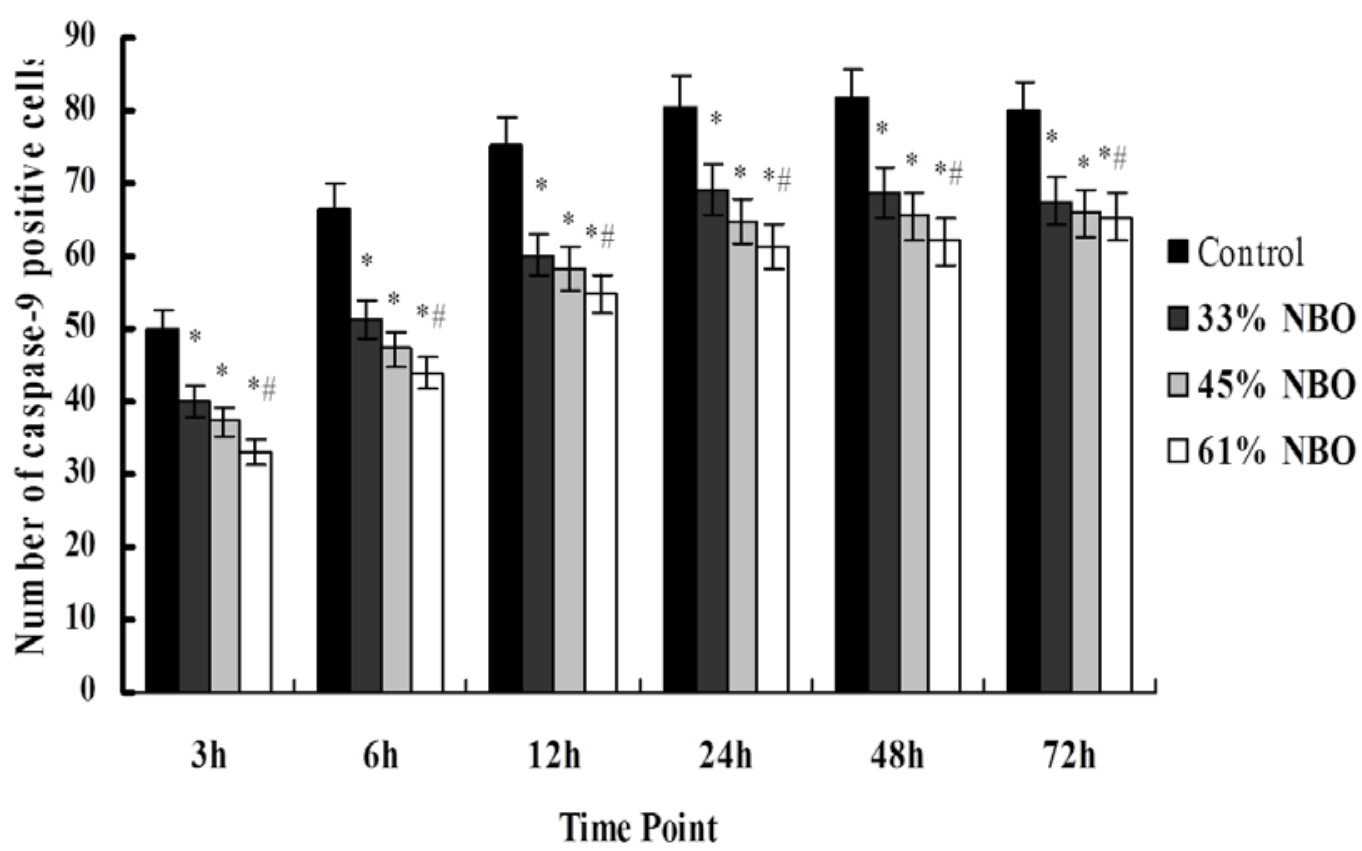

